University of Nebraska - Lincoln

DigitalCommons@University of Nebraska - Lincoln

Uniformed Services University of the Health

Sciences

U.S. Department of Defense

2011

\title{
Safety and immunogenicity of an intranasal Shigella flexneri 2a Invaplex 50 vaccine
}

\author{
Mark S. Riddle \\ Naval Medical Research Center \\ Robert W. Kaminski \\ Walter Reed Army Institute of Research \\ Carlos Williams \\ Naval Medical Research Center \\ Chad Porter \\ Naval Medical Research Center \\ Shahida Baqar \\ Naval Medical Research Center \\ See next page for additional authors \\ Follow this and additional works at: https://digitalcommons.unl.edu/usuhs \\ Part of the Medicine and Health Sciences Commons
}

Riddle, Mark S.; Kaminski, Robert W.; Williams, Carlos; Porter, Chad; Baqar, Shahida; Kordis, Alexis; Gilliland, Theron; Lapa, Joyce; Coughlin, Melissa; Soltis, Chris; Jones, Erica; Saunders, Jackie; Keiser, Paul B.; Ranallo, Ryan T.; Gormley, Robert; Nelson, Michael; Turbyfill, K. Ross; Tribble, David; and Oaks, Edwin V., "Safety and immunogenicity of an intranasal Shigella flexneri 2a Invaplex 50 vaccine" (2011). Uniformed Services University of the Health Sciences. 95.

https://digitalcommons.unl.edu/usuhs/95

This Article is brought to you for free and open access by the U.S. Department of Defense at DigitalCommons@University of Nebraska - Lincoln. It has been accepted for inclusion in Uniformed Services University of the Health Sciences by an authorized administrator of DigitalCommons@University of Nebraska Lincoln. 


\section{Authors}

Mark S. Riddle, Robert W. Kaminski, Carlos Williams, Chad Porter, Shahida Baqar, Alexis Kordis, Theron Gilliland, Joyce Lapa, Melissa Coughlin, Chris Soltis, Erica Jones, Jackie Saunders, Paul B. Keiser, Ryan T. Ranallo, Robert Gormley, Michael Nelson, K. Ross Turbyfill, David Tribble, and Edwin V. Oaks 


\title{
Safety and immunogenicity of an intranasal Shigella flexneri 2a Invaplex 50 vaccine $^{\text {is }}$
}

\author{
Mark S. Riddle ${ }^{\mathrm{a}}$, Robert W. Kaminski ${ }^{\mathrm{b}}$, Carlos Williams ${ }^{\mathrm{a}}$, Chad Porter ${ }^{\mathrm{a}}$, Shahida Baqar ${ }^{\mathrm{a}, 1}$, \\ Alexis Kordis ${ }^{\mathrm{b}}$, Theron Gilliland ${ }^{\mathrm{a}}$, Joyce Lapa ${ }^{\mathrm{a}}$, Melissa Coughlin ${ }^{\mathrm{b}}$, Chris Soltis ${ }^{\mathrm{c}}$, \\ Erica Jones $^{\text {b }}$, Jackie Saunders ${ }^{\mathrm{b}}$, Paul B. Keiser ${ }^{\mathrm{b}, \mathrm{c}}$, Ryan T. Ranallo ${ }^{\mathrm{b}}$, Robert Gormley ${ }^{\mathrm{a}}$, \\ Michael Nelson $^{c}$, K. Ross Turbyfill ${ }^{\mathrm{b}}$, David Tribble ${ }^{\mathrm{d}}$, Edwin V. Oaks ${ }^{\mathrm{b}, *}$ \\ a Naval Medical Research Center, Silver Spring, MD, United States \\ ${ }^{\mathrm{b}}$ Walter Reed Army Institute of Research, Silver Spring, MD, United States \\ ${ }^{c}$ Walter Reed Army Medical Center, Washington, DC, United States \\ ${ }^{\mathrm{d}}$ Uniformed Services University of the Health Sciences, Bethesda, MD, United States
}

\section{A R T I C L E I N F O}

\section{Article history:}

Received 27 May 2011

Received in revised form 7 July 2011

Accepted 11 July 2011

Available online 23 July 2011

\section{Keywords:}

Shigella flexneri

Invaplex

Nasal vaccine

Immunogenicity

\begin{abstract}
A B S T R A C T
Background: Shigella flexneri 2a lipopolysaccharide 50 is a nasally delivered subunit vaccine consisting of a macromolecular complex composed of LPS, IpaB, IpaC and IpaD. The current study examined vaccine safety and immunogenicity across a dose range and the clinical performance of a new intranasal delivery device.

Methods: Volunteers $(N=36)$ were randomized to receive vaccine via the Dolphin ${ }^{\mathrm{TM}}$ (Valois of America, Congers, New York) intranasal spray device at one of three doses (240, 480, and $690 \mu \mathrm{g})$ on days 0,14 , and 28. Another group $(N=8)$ received the $240 \mu \mathrm{g}$ dose via pipette. Vaccine safety was actively monitored and antigen-specific humoral and mucosal immune responses were determined.

Results: There were no serious adverse events and the majority of adverse events (98\%) were mild. Antibody secreting cells (ASC), plasma, and mucosal immune responses to Shigella antigens were detected at all three dose levels with the $690 \mu \mathrm{g}$ dose inducing the highest magnitude and frequency of responses. Vaccination with comparable doses of Invaplex 50 via the Dolphin ${ }^{\mathrm{TM}}$ resulted in higher plasma and ASC immune responses as compared to pipette delivery.

Conclusion: In this trial the S. flexneri 2a Invaplex 50 vaccine was safe, well-tolerated and induced robust levels of antigen-specific intestinal IgA and ASC responses. The spray device performed well and offered an advantage over pipette intranasal delivery.
\end{abstract}

Published by Elsevier Ltd.

\section{Background}

Shigellosis is a leading cause of diarrheal disease worldwide particularly in developing countries [1], as well as a continu-

\footnotetext{
The views expressed in this article are those of the author and do not necessarily reflect the official policy or position of the Department of the Navy, Department of the Army, Department of Defense, nor the U.S. Government. The mention of trade names, commercial products, or organizations does not imply endorsement by the U.S. government. The NIAID affiliation of SB is for identification purposes only; NIAID does not endorse the research findings.

* Corresponding author at: Division of Bacterial and Rickettsial Diseases, Subunit Enteric Vaccines and Immunology, Walter Reed Army Institute of Research, 503 Robert Grant Avenue, Silver Spring, MD 20910-7500, United States. Tel.: +1 301319 9268; fax: +1 3013199801 .

E-mail address: edwin.oaks@us.army.mil (E.V. Oaks).

1 Current address: Division of Microbiology and Infectious Diseases, National Institute of Allergy and Infectious Diseases, National Institutes of Health, Bethesda, MD 20892, United States.
}

ing problem for civilian travelers and military visiting endemic regions [2-5]. Vaccine development remains a high priority given the disease burden, increasing antibiotic resistance, and growing appreciation of post-infectious sequelae associated with shigellosis [6,7]. Shigella flexneri accounts for $30-60 \%$ of shigellosis cases in developing regions necessitating coverage of prevalent $S$. flexneri serotypes in a multivalent Shigella vaccine [1].

Several vaccine approaches to prevent shigellosis are under active investigation including live-attenuated vaccines, inactivated whole cell vaccines, subcellular vaccines and purified subunit vaccines such as $\mathrm{O}$-specific polysaccharide conjugate vaccines $[8,9]$. The lack of a clear correlate of protection for Shigella vaccines has hampered vaccine development over the past several decades $[8,10,11]$. Even so, the importance of the serotype specific LPS antigen is widely recognized and included as a component of all vaccine approaches actively being pursued. Protein antigens, such as the Ipa protein effectors of the type three-secretion system, remain attractive vaccine candidates due to their active role in pathogen-directed 
cellular invasion and the highly conserved sequences among these essential virulence factors [12,13]. An effective Shigella vaccine must deliver the appropriate immunogens and also stimulate the proper immune response phenotype. For shigellosis, this likely consists of local immunity within the intestinal tract. Oral immunization may achieve intestinal immunity but is often difficult to consistently accomplish due to delivery restrictions, particularly for inactivated or subunit vaccines. Mucosal immune responses can also be achieved with intranasal immunization, although it is not clear if this is an effective route for stimulating intestinal immune responses in humans. The Shigella Invaplex 50 vaccine, currently under clinical investigation, is a macromolecular complex isolated from wild-type $S$. flexneri 2a that included both serotype specific (LPS) and conserved antigens (IpaB, IpaC and IpaD) and upon intranasal immunization stimulates robust intestinal and pulmonary immune responses [14-16].

An initial phase 1 dose-escalation study evaluating the safety and immunogenicity of the $S$. flexneri Invaplex 50 vaccine produced under current Good Manufacturing Practices (cGMP) was recently conducted using a pipette to deliver the vaccine intranasally [16]. The Invaplex 50 vaccine was well tolerated and resulted in antigen-specific humoral and mucosal immune responses at doses $\geq 240 \mu \mathrm{g}$. In the current study, the S. flexneri 2a Invaplex 50 vaccine was evaluated using a new lot of cGMP Invaplex 50 to expand the dose range and safety monitoring. Additionally, a nasal delivery device was evaluated to facilitate vaccine delivery and potentially induce a more robust mucosal immune response due to distribution of the vaccine over a greater mucosal surface as compared to pipette delivery.

\section{Materials and methods}

\subsection{Manufacture of Invaplex 50 vaccine}

The cGMP Invaplex 50 vaccine was prepared from virulent S. flexneri $2 \mathrm{a}$, strain $2457 \mathrm{~T}$ as previously described $[14,16]$ with the following modifications. The 3001 culture was incubated at $37^{\circ} \mathrm{C}$ with an agitation speed of $200 \mathrm{rpm}$ and air flow of $1501 / \mathrm{min}$, in animal product-free modified Antibiotic Medium 3 with $0.003 \%$ antifoam. The animal product-free modified Antibiotic Medium 3 contained the following components per liter: Bacto yeast extract (Becton Dickinson, Sparks, MD), $1.5 \mathrm{~g}$; Vegetable Peptone No. 1 (Oxoid/Remel, Lenexa, KS), 5 g; Bacto Dextrose (Difco/BD, Sparks, MD) $1 \mathrm{~g}$; sodium chloride (molecular-biology tested, Sigma-Aldrich, St. Louis, MO), $3.5 \mathrm{~g}$; potassium phosphate, dibasic (Sigma-Aldrich), $3.68 \mathrm{~g}$; and, potassium phosphate, monobasic (Sigma-Aldrich), $1.32 \mathrm{~g}$. After $18 \mathrm{~h}$ of growth, the culture was harvested by centrifugation (Sharples AS-26 continuous feed centrifuge) and an aliquot of the final culture was used for determination of bacterial colony forming units ( $\mathrm{fu}$ ), gram stain, purity, colony uniformity, percent Congo red positive colonies and organism identity. The collected cells were extracted with water and the resulting extract was applied to an anion-exchange column (Q Sepharose High Performance, GE Healthcare) for isolation of the Invaplex 50 product in an elution step consisting of $500 \mathrm{mM}$ $\mathrm{NaCl}$ in $20 \mathrm{mM}$ Tris, pH 9.0 as previously described [16]. The final Invaplex 50 product was adjusted to $250 \mathrm{mM} \mathrm{NaCl}$ in $20 \mathrm{mM}$ Tris, $\mathrm{pH}$ 8.8 and a final protein concentration of $3.46 \mathrm{mg}$ protein $/ \mathrm{ml}$, sterilized by filtration $(0.22 \mu \mathrm{m}$ Millipak-20 filter unit) and dispensed in $1.0 \mathrm{ml}$ volumes into sterile, depyrogenated $2 \mathrm{ml}$ glass vials without preservative. All vials were stored at $-80^{\circ} \mathrm{C}$.

\subsection{Analysis of cGMP S. flexneri 2a Invaplex 50 lot 1307}

The total protein concentration was measured by the BCA (bicinchoninic acid) assay (BioRad). SDS-PAGE Coomassie blue-stained gels, silver-stained gels and western blots using anti-IpaB, IpaC and S. flexneri 2a LPS mAbs $[17,18]$ were used to assess the protein and LPS composition in the Invaplex 50 product. The amount of IpaB and IpaC in Invaplex 50 was determined using a modified ELISA procedure [19]. The LAL (Limulus amebocyte lysate) gel clot method (Pryotell, Associates of Cape Cod, Inc.) was used to measure the quantity of lipopolysaccharide [16]. For the various analyses, the animal product free-derived Invaplex 50 lot 1307 was compared to previous cGMP lots 0994 and 0808 that were prepared from $S$. flexneri 2a grown in Antibiotic Medium 3 containing animal derived products [16].

\subsection{Immunogenicity and efficacy in small animals}

The immunogenicity and efficacy of the cGMP S. flexneri 2a Invaplex 50 lot 1307 vaccine was evaluated in mice and guinea pigs. These procedures have been previously described for other lots of cGMP Invaplex 50 [16].

\subsection{Stability of Invaplex 50 vaccine}

The Invaplex 50 product lot 1307 , stored at $-80^{\circ} \mathrm{C}$, was assessed annually for antigen content by quantitative and qualitative assays described above and immunogenicity in guinea pigs. At the time of the study described in this report, lot 1307 had undergone three immunogenicity evaluations at 4,12 and 20 months and the serum antibody response to S. flexneri 2a LPS and Invaplex 50 was evaluated.

\subsection{Stability of Invaplex 50 in the Dolphin ${ }^{\mathrm{TM}}$ device}

To improve the distribution of antigen on the nasal mucosa and enhance antigen uptake, the Dolphin ${ }^{\mathrm{TM}}$ system (Valois of America, Congers, NY), a single use prefillable, dispensing device was used. The Dolphin ${ }^{\mathrm{TM}}$ ensures reproducible droplet distribution on the nasal mucosa with a consistent size of $\sim 100 \mu \mathrm{m}$ which is more effective for antigen uptake by $\mathrm{M}$ cells, or antigen delivery to antigen presenting cells (APCs) of the mucosal immune system [20]. The Dolphin $^{\mathrm{TM}}$ intranasal spray device has not been used previously to deliver vaccine products. A study was conducted to evaluate the stability of the Invaplex 50 lot 1307 in the Dolphin ${ }^{\mathrm{TM}}$ device under conditions that simulated the product formulation and storage in the device on the day of immunization. Multiple devices were loaded with Invaplex 50 vaccine $(230 \mu \mathrm{l}, 2.4 \mathrm{mg}$ protein $/ \mathrm{ml})$ and placed at $4{ }^{\circ} \mathrm{C}$. At $0,2,4,5$ and $6 \mathrm{~h}$ a device was removed from the refrigerator and discharged twice ( $100 \mu \mathrm{l}$ per spray) into a $50 \mathrm{ml}$ conical tube to collect the $200 \mu$ l dose volume (spray). The dead volume of the device is approximately $30 \mu$ l. The sprayed vaccine was centrifuged ( $2000 \mathrm{rpm}, 5 \mathrm{~min}, 4^{\circ} \mathrm{C}$ ), collected and stored at $-80^{\circ} \mathrm{C}$. Prior to and following the discharge event the Dolphin device was weighed to estimate the total volume discharged. Similar studies to measure reproducibility of the spray volumes were also conducted with saline. Collected samples were evaluated for total protein concentration by the BCA protein assay and the stability of IpaB, IpaC and LPS by western blots (IpaB and IpaC) and silver-stained gels (LPS). The relative content of LPS, IpaB and IpaC at each time point (as compared to untreated sample) was determined by densitometry analysis of the bands in silver-stained gels or western blots.

\subsection{Clinical trial design}

The study was conducted as an outpatient, single center, randomized, double-blind study in 36 healthy adult volunteers to assess vaccine safety and immunogenicity. Volunteers (12 per group) received one of three intranasal dose amounts (240, 480 or $690 \mu \mathrm{g}$ ) by the Dolphin ${ }^{\mathrm{TM}}$ device. In addition, a fourth 
group of 8 volunteers received a $240 \mu \mathrm{g}$ intranasal dose delivered by pipette to serve as a bridging group to the preceding trial [16].

\subsection{Study population and enrollment criteria}

Volunteers were healthy male and non-pregnant female adults between 18 and 45 years of age recruited from the Baltimore, MD-Washington, DC greater metropolitan area. They were enrolled after an informed consent process consisting of a detailed presentation of study material via a taped video, a comprehension test, and interview with an investigator. Volunteers were excluded from enrollment if they had clinically significant acute or chronic diseases, immunosuppressive disorders or medication, regularly used antidiarrheal, anti-constipation, or antacid therapy, an abnormal stool pattern ( $<3$ stools per week, or $>3$ stools per day), had participated in other investigational product research, had a positive blood test for Hepatitis B surface antigen, antibodies to Hepatitis $C$ virus or Human Immunodeficiency Virus-1, or had clinically significant abnormalities on basic laboratory screening. Furthermore, given the intranasal route of administration, volunteers were excluded if they had nasal polyps, ulcers of the nasal mucosa, or clinically significant nasal septal deviation, or a past/current medical history of chronic sinusitis or chronic/seasonal rhinitis, rhinoplasty, reactive airway disease, chronic obstructive pulmonary disease, chronic bronchitis, Bell's palsy, or were a current or recent ( $\leq 3$ months) smoker. Based on epidemiological data and the identification of Shigella LPS isolated in the synovial fluid in reactive arthritis [21,22], volunteers with a personal or family history of an inflammatory arthritis or positive blood test for HLA-B27 were excluded. Lastly, volunteers with a history of microbiologically confirmed Shigella infection, prior receipt of an experimental Shigella vaccine or live Shigella challenge, recent travel ( $\leq 2$ years) to a country where Shigella or other enteric infections are endemic, recent ( $\leq 3$ years) occupation involving exposure to Shigella species, or a plasma IgG titer $\geq 2500$ to S. flexneri 2a LPS were excluded.

\subsection{Randomization, concealment and immunization procedures}

Participants were randomized to one of three dose cohorts using a ratio of 1:1:1 in block sizes of 3 . The group receiving the Invaplex 50 vaccine via pipette was not randomized. A coding system was used so that clinical investigators could not ascertain group assignment. The vaccine was a clear liquid and did not differ visually among the different dose levels. Depending on group assignment, the volunteer received either 240,480 or $690 \mu \mathrm{g}$ of vaccine in a fixed volume of $200 \mu \mathrm{l}$ from two consecutive sprays (approximately $100 \mu \mathrm{l} /$ spray per nostril). To avoid anterior and posterior dripping of the vaccine formulation out of the nose or into the digestive tract, volunteers were vaccinated in a sitting position on an exam table, with their head in a neutral or slightly tilted forward position. The volunteer was instructed to breathe normally in through the nose and out through the mouth, whereby a trained investigator delivered the spray during inspiration. For delivery the device was aimed at the medial angle of the eye (away from nasal septum) and with the device kept at an angle less than $45^{\circ}$ from vertical. After each spray the subject was reclined $\left(\leq 45^{\circ}\right)$ onto an exam table with the head in neutral to slightly tilted back position, where the subject was instructed to continue breathing gently for 30-60 s. Nasal sprays were given consecutively after an intervening delay. Volunteers received $240 \mu \mathrm{g}$ of Invaplex 50 via nasal pipette in a total volume of $200 \mu \mathrm{l}$ (100 $\mu \mathrm{l}$ per nostril) as previously described [16]. Three doses of the vaccine were given at two-week intervals to all subjects.

\subsection{Safety monitoring}

Adverse event monitoring was conducted using in-person symptom surveillance, symptom diary log, and targeted physical exams. Specifically, baseline clinical assessments including symptom survey and local physical exam findings were conducted at screening (up to 90 days prior to vaccination), 7 days prior to immunization and on the day of immunization to record preimmunization findings. After each immunization, volunteers were observed for $30 \mathrm{~min}$ immediately followed by a symptom survey and local physical exam. Adverse events were recorded prior to and 30 min after each immunization. Twice daily, for 7 days postvaccination, volunteers documented all adverse events in a diary. Volunteers returned to the clinic 7 days after each immunization for a focused exam and a diary review. The clinical observation period concluded 28 days after the third vaccine dose. Solicited adverse events included malaise, headache, rhinorrhea, nasal congestion, nasal burning, nasal itching, sore throat, postnasal drip, cough, sinus pain, sneezing, itching eyes, epistaxis and fever. Information on any other symptoms, the use of any medications (prescription and/or over-the-counter), non-planned medical consultations or doctor's visits, and hospitalizations were also collected. Severity of self-reported symptoms (adverse events) were recorded according to the following grading scale: absent (Grade 0 ), mild (Grade $1 \mathrm{a}=$ barely noticeable, Grade $1 \mathrm{~b}=$ noticeable, but not interfering with daily activities), moderate (Grade $2=$ interfering with daily activities), severe (Grade 3 = preventing daily activities), and serious (Grade 4 =fatal or life-threatening, causing a prolonged hospitalization, resulting in a significant, persistent, or permanent disability, or requiring intervention to prevent permanent impairment or damage). In addition to self-reporting of nasal adverse reactions, standardized physical examinations with visualization of the nasal mucosa, septum and mucosal blood vessel status after the administration of each dose of the intranasal vaccine were conducted by trained physicians (predominately by a single allergist/immunologist). Examinations included an assessment for nasal mucosa hyperemia, nasal discharge, nasal edema, pharyngeal erythema, sinus tenderness, lymphadenopathy, conjunctival injection, tearing, epistaxis, abnormal lung exam finding, abnormal cranial nerve finding, and were similarly coded as mild, moderate, severe and serious based on previously used grading criteria [16]. Blood for clinical safety assessment was drawn 7 days following the third vaccine dose. In addition to grading severity of adverse events, the degree of certainty (definite, probable, possible and unrelated) with which an adverse event could be attributed to administration of the test article was determined by the principal investigator. The recording of adverse events was solicited by a trained physician, with assignment of relationship made only by the principal investigator. All aspects of the trial were closely assessed by an independent medical monitor. Established criteria for discontinuation of test article administration for all volunteers included any individual experiencing any serious adverse events related to the Invaplex 50 product and/or the occurrence of two unexpected, severe adverse events related to the Invaplex 50 product.

\subsection{Sample collections, schedules, and processing}

The following samples were collected for immunological analysis: plasma for antigen-specific IgG and IgA (days 0, 14, 28, 35, 42, and 56), peripheral blood mononuclear cells (PBMCs) for antigenspecific antibody secreting cells (days 0,21 , and 35 ), stool (days $0,21,35$, and 56), and nasal washes (day $-7,21,35$, and 56) for secretory IgA. Blood samples were separated into plasma and PBMC fractions as previously described [16]. Stool and nasal specimens were frozen $\left(-70^{\circ} \mathrm{C}\right)$ immediately after collection and $\operatorname{IgA}$ was extracted as described [16]. 


\subsection{Determination of antibody titers in plasma and mucosal samples}

The Shigella antigens, including S. flexneri 2a LPS and S. flexneri 2a Invaplex 50, used to measure the vaccine-specific immune response have been previously described [16]. The $S$. flexneri $2 \mathrm{a}$ Invaplex 50 was a research grade lot of Invaplex 50 that had similar antigen content as the cGMP lot used for immunization of the volunteers. For immunoassays, antigen plates were coated overnight at $4{ }^{\circ} \mathrm{C}$ with $100 \mu \mathrm{l}$ of LPS or Invaplex 50 diluted to 10 or $0.5 \mu \mathrm{g} / \mathrm{ml}$, respectively, in carbonate coating buffer ( $\mathrm{pH} 9.8$ ). IgG and IgA endpoint titers specific for Shigella antigens were determined by ELISA from plasma samples as previously described [16]. The endpoint titer was defined as the reciprocal of the last dilution of a given sample that produced an $\mathrm{OD}_{405}$ value of $\geq 0.2$.

Nasal and fecal samples were assessed by ELISA for anti-S. flexneri 2a LPS and anti-S. flexneri 2a Invaplex 50 IgA titers as previously described $[16,23]$ with minor modifications. First, total IgA in stool extracts and nasal extracts was determined by a capture ELISA [24] using goat anti-human $\mathrm{F}\left(\mathrm{ab}^{\prime}\right)_{2}$ (Jackson Laboratories) as capture antibody and HRP conjugated goat anti-human IgA (KPL, Rockville, MD) as the detecting antibody. Total IgA concentrations were then adjusted to $1 \mathrm{mg} / \mathrm{ml}$ by either concentration with Amicon Ultra 30,000 MWCO filters (Millipore, Billerica, MA) or dilution and antigen-specific endpoint titers were determined as outlined above. Endpoint titers of mucosal specimens were calculated as the reciprocal of the highest dilution giving a net (antigen wells-BSA coated control wells) absorbance value of $\geq 0.15$.

\subsection{Antibody secreting cell (ASC) assay}

$\operatorname{IgA}$ and IgG ASCs specific for S. flexneri 2a LPS and S. flexneri 2a Invaplex 50 were enumerated by an ASC assay using samples collected on days 0,21 , and 35 as previously described [16] using S. flexneri 2a LPS, Invaplex 50, and BSA diluted to $10,0.5$, or $1 \mu \mathrm{g} / \mathrm{ml}$ (100 $\mu \mathrm{l} /$ well), respectively, in carbonate coating buffer ( $\mathrm{pH} 9.8$ ).

\subsection{Study endpoints and definitions}

The primary safety outcome was local or systemic reactions occurring in the 7-day post-vaccination period. A priori immunological outcomes for responders were established as follows: (1) ASC positive response: $\geq 10$ antigen-specific ASC per $10^{6}$ PBMCs; (2) seroconversion: $\geq 4$-fold increase over the baseline titer; and (3) fecal and nasal IgA responders: $\geq 4$-fold increase over the baseline titer after normalizing for total IgA. Only volunteers that received at least two doses of vaccine were included in the immunological analysis.

\subsection{Data analysis and statistical considerations}

Rates of all adverse events (related and unrelated) observed during the follow-up period after vaccinations were analyzed to compare within and across dose levels. For immunological analysis, qualitative (responder rates) and quantitative assessments (log transformed values) were made in addition to evaluation of the kinetics of the immune response. Nonparametric tests were used for comparison between groups (Kruskal-Wallis for continuous data and Fisher's exact test for categorical data). Paired $t$-tests were used to compare individual post-vaccination to baseline response within each treatment group. All statistical tests were interpreted in a two-tailed fashion using alpha $\leq 0.05$; no adjustments were made for multiple comparisons.

As this was a phase 1 dose-finding clinical trial, no formal sample size calculation was performed; however, each treatment group sample size (minimum $n=10$, with exception of bridging pipette group) ensured that the probability of detecting at least one adverse event in the group was $80 \%$, provided that the true adverse event rate exceeded 15\%. Furthermore, using a binomial probability formula for no observed severe adverse events within the 36 volunteers receiving at least one dose of test article via the Dolphin $^{\mathrm{TM}}$ device yielded a $95 \%$ confidence interval of $0-11 \%$.

\section{Results}

\subsection{Characteristics of the Invaplex 50 vaccine lot 1307}

The composition of S. flexneri 2a Invaplex 50 lot 1307 was comparable to Invaplex 50 lot 0994 that was used in the initial phase 1 study [16]. Invaplex 50 lot 1307 contained $12.4 \mu \mathrm{g} \mathrm{IpaB} / \mathrm{mg}$ total protein, $18.8 \mu \mathrm{g} \mathrm{IpaC} / \mathrm{mg}$ total protein and $6 \times 10^{6} \mathrm{EU} / \mathrm{ml}$ (567 $\mu \mathrm{g}$ LPS $/ \mathrm{ml}$ ) or $1.7 \times 10^{6} \mathrm{EU} / \mathrm{mg}$ total protein $(164 \mu \mathrm{g} \mathrm{LPS} / \mathrm{mg}$ total protein). For comparison, lot 0994 contained $8.4 \mu \mathrm{g} \mathrm{IpaB} / \mathrm{mg}$ total protein, $18.1 \mu \mathrm{g} \mathrm{IpaC} / \mathrm{mg}$ total protein and $1.0 \times 10^{6} \mathrm{EU} / \mathrm{mg}$ total protein [16]. At the time of manufacture, the immunogenicity and efficacy of lot 1307 was assessed in mice and guinea pigs (Table 1). Using S. flexneri 2a LPS and research grade Invaplex 50 as ELISA antigens, S. flexneri 2a Invaplex 50 lot 1307 stimulated a greater than 16 -fold increase $(p<0.001)$ in titer in all immunized mice and a greater than 8 -fold increase $(p<0.001)$ in all immunized guinea pigs as compared to the saline control group. At the time of manufacture lot 1307 provided protective immunity in both mice and guinea pigs. In mice, using the lethal lung model, 15 of 15 challenged mice were protected $(p<0.0001$; control mice 11 of 13 mice died). In guinea pigs, using the keratoconjunctivitis model, $83 \%(n=12)$ of the animals were protected $(p<0.001 ; 100 \%$ saline controls $(n=10)$ were infected with scores of 3$)$. The level of protection achieved with lot 1307 (made from cultures grown in animal product-free media) was comparable to previously described cGMP Invaplex 50 lots 0994 and 0808 made from S. flexneri 2a grown in animal product-containing media. Immunogenicity assessments were also conducted periodically on the product as part of the stability program and showed comparable titers at 12 months and 20 months post-manufacture (data not shown).

\subsection{Stability of S. flexneri 2a Invaplex 50 lot 1307 vaccine}

At the time of the clinical trial described in this manuscript the IpaB, IpaC and LPS antigens in lot 1307 showed no evidence of degradation or decreased quantities as determined by western blots and SDS-PAGE. Similarly the immunogenicity in guinea pigs at the time of the trial was comparable to that determined at the time of manufacture. Serum IgG titers specific for LPS and Invaplex 20 months post-manufacture were comparable in magnitude (GMT 1091 and 3800, respectively) to those induced after vaccination 4 months post-manufacture (GMT 1440 and 2036, respectively) with all animals responding to vaccination with a greater than or equal to 4 -fold increase in antibody titers over baseline (day 0). Similar trends were evident in the serum IgA titers, with comparable levels of anti-Invaplex 50 and anti-LPS antibodies induced after vaccination with product stored at $-80^{\circ} \mathrm{C}$ for up to 20 months.

The stability of the Invaplex 50 vaccine in the Dolphin ${ }^{\mathrm{TM}}$ device was determined under conditions comparable to those used for immunizations in the clinic. The quantity of each antigen was consistent over the $6 \mathrm{~h}$ time course and increased degradation of IpaB and IpaC was not detected by densitometry western blot analysis using untreated vaccine as the baseline. The LPS content ranged from 86 to $130 \%$ of the untreated value, IpaB content ranged from 85 to $135 \%$, and IpaC content ranged from 88 to $111 \%$. This suggests that the Invaplex 50 product was stable when stored in a Dolphin 
Table 1

Results of efficacy experiments in mice ${ }^{\mathrm{a}}$ and guinea pigs ${ }^{\mathrm{b}}$ with after intranasal immunization with S. flexneri 2a Invaplex 50 lots 1307,0994 and 0808.

\begin{tabular}{|c|c|c|c|c|c|c|c|}
\hline Animal model & Vaccine $^{\mathrm{c}}$ (dose) & $\begin{array}{l}\text { Anti-S. flexneri } 2 \mathrm{a} \\
\text { LPS serum IgG }\end{array}$ & $\begin{array}{l}\text { Anti-S. flexneri } 2 \mathrm{a} \\
\text { Invaplex serum IgG }\end{array}$ & $\begin{array}{l}\text { Number deaths/ } \\
\text { total }\end{array}$ & $\%$ death & Efficacy ${ }^{\mathrm{e}}$ & $p$-Value ${ }^{\mathrm{f}}$ \\
\hline \multirow[t]{4}{*}{ Mouse lung } & Invaplex 50 lot $1307(5 \mu \mathrm{g})$ & $360 \pm 531^{d}$ & $6617 \pm 3864$ & $0 / 15$ & 0 & $100 \%$ & $<0.001$ \\
\hline & Invaplex 50 lot $0994(5 \mu \mathrm{g})$ & $360 \pm 531$ & $3800 \pm 2036$ & $0 / 15$ & 0 & $100 \%$ & $<0.001$ \\
\hline & Invaplex 50 lot $0808(5 \mu \mathrm{g})$ & $207 \pm 121$ & $5014 \pm 3527$ & $1 / 15$ & 7 & $92 \%$ & $<0.001$ \\
\hline & Saline & $90 \pm 0$ & $90 \pm 0$ & $11 / 13$ & 85 & - & - \\
\hline Animal model & Vaccine $^{c}$ (dose) & $\begin{array}{l}\text { Anti-S. flexneri 2a } \\
\text { LPS serum IgG }\end{array}$ & $\begin{array}{l}\text { Anti-S. flexneri } 2 \mathrm{a} \\
\text { Invaplex serum IgG }\end{array}$ & $\begin{array}{l}\text { Number with } \\
\text { disease/total }\end{array}$ & $\%$ disease & Efficacy & $p$-Value ${ }^{\mathrm{f}}$ \\
\hline Guinea pig & Invaplex 50 lot $1307(25 \mu \mathrm{g})$ & $1440 \pm 2556$ & $2036 \pm 789$ & $2 / 12$ & 17 & $83 \%$ & $<0.001$ \\
\hline \multirow[t]{3}{*}{ keratoconjunctivitis } & Invaplex 50 lot $0994(25 \mu \mathrm{g})$ & $1440 \pm 984$ & $2286 \pm 1683$ & $4 / 12$ & 33 & $67 \%$ & 0.002 \\
\hline & Invaplex 50 lot $0808(25 \mu \mathrm{g})$ & $360 \pm 511$ & $2286 \pm 2485$ & $4 / 12$ & 33 & $67 \%$ & 0.002 \\
\hline & Saline & $90 \pm 0$ & $90 \pm 0$ & $10 / 10$ & 100 & - & - \\
\hline
\end{tabular}

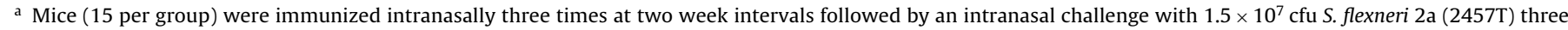
weeks after the final immunization. Deaths (endpoint) were recorded daily for two weeks after challenge.

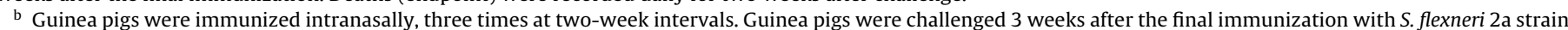

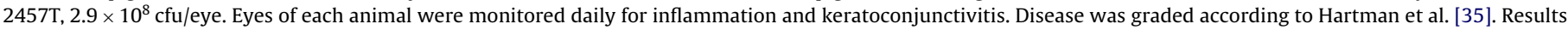
on day 5 were used to determine protection. Scores of 2 and 3 were considered positive for disease.

c Invaplex 50 lots 0994 and 0808 are described in Tribble et al. [16]

d Geometric mean titer (GMT) \pm standard deviation.

e Efficacy was calculated by the formula: [ $\{\%$ disease or death (controls) $-\%$ disease or death (vaccines) $\} / \%$ disease or death (controls)] $\times 100$.

${ }^{\mathrm{f}} p$-Value was determined by the Fisher's exact test.

nasal spray device at $4{ }^{\circ} \mathrm{C}$ for up to $6 \mathrm{~h}$. For clinical use, a 5-h limitation was applied for the Invaplex 50 product stored in the device at $4{ }^{\circ} \mathrm{C}$

\subsection{Dolphin ${ }^{\mathrm{TM}}$ device spray reproducibility}

The Dolphin ${ }^{\mathrm{TM}}$ is a unit-spray device (Fig. 1) designed to deliver $200 \mu \mathrm{l}$ of product in two, $100 \mu \mathrm{l}$ sprays. The manufacturer (Valois of America, Inc.) has determined that the mean delivery volume is $200.1 \mu \mathrm{l}$ with a range of $193.9-207.5 \mu \mathrm{l}$. Less than $1 \%$ of the spray droplets from each spray are smaller than $10 \mu \mathrm{m}$ in diameter which reduces the probability of the sprayed product entering the lungs. The average diameter of the droplets is $100.9 \pm 11.5 \mu \mathrm{m}$ for the first spray and $81.7 \pm 10.4 \mu \mathrm{m}$ for the second spray. In the Walter Reed Army Institute of Research (WRAIR) laboratory the mean spray volume for saline was $196.9 \pm 4.3 \mu \mathrm{l}$ and for Invaplex 50 it was $194.9 \pm 10.1 \mu \mathrm{l}$. During clinical trials it was not possible to determine the actual volume sprayed but the amount retained in the device was determined for each of the three dose amounts of Invaplex 50 given during the trial (Table 2). Although the retained volume ranged from $19.7 \mu \mathrm{l}$ to a maximum of $77.1 \mu$ l there was no

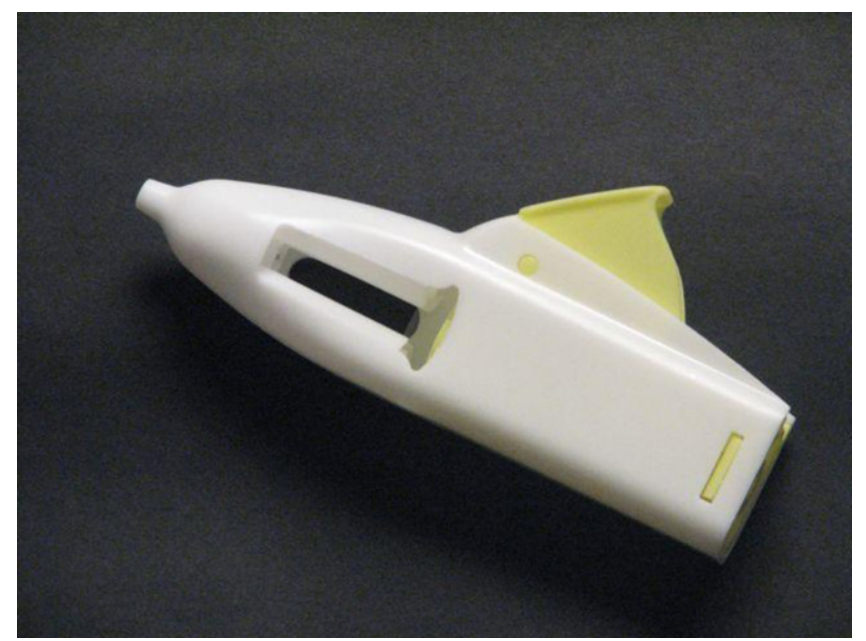

Fig. 1. The Dolphin ${ }^{\mathrm{TM}}$ unit-spray device. (Valois of America, Inc.) significant difference in the retained volumes between the three dose groups.

\subsection{Clinical trial results}

\subsubsection{Demographics}

A total of 120 volunteers initiated the informed consent process and 112 signed a written informed consent and underwent pre-study screening (Fig. 2). Reasons for individuals (44 subjects) not meeting eligibility criteria included disqualifying medical history or physical exam ( $n=6,14 \%)$, clinically significant, persistent abnormal baseline biochemistry or hematology tests $(n=15,34 \%)$, positive blood test for HBsAG or antibodies to HCV or HIV-1 ( $n=4$, $9 \%)$, history of reactive arthritis $(n=1,2 \%)$, and positive blood test for HLA-B27 ( $n=5,11 \%)$, current smoker $(n=1,2 \%)$, serum IgG titer to Shigella LPS $\geq 2500(n=4,9 \%)$, and recent travel to Shigella endemic region $(n=8,18 \%)$. Among the remaining 44 enrolled participants, 36 were randomized to the three cohorts immunized with the Dolphin device and received at least one dose of study vaccine. Eight other enrolled participants were administered $240 \mu \mathrm{g}$ vaccine with a pipette. The mean age of participants was 36.1 years (range 19-45 years). While there were no significant differences in the gender ( $52.8 \%$ male) or race (66.7\% African-American) between vaccine groups, the $690 \mu \mathrm{g}$ dose group was younger $(p=0.002)$ than the 240 or $480 \mu \mathrm{g}$ dose groups (Table 3 ).

\subsubsection{Safety and clinical adverse events}

There were no serious adverse events or adverse events that met stopping criteria. Additionally, there were no vaccineassociated, clinically significant abnormalities in hematology or

Table 2

Retained S. flexneri 2a Invaplex 50 volume in Dolphin ${ }^{\mathrm{TM}}$ device after vaccine administration.

\begin{tabular}{llll}
\hline & \multicolumn{3}{l}{ Dose amount $(\mu \mathrm{g})$} \\
\cline { 2 - 4 } & $240 \mu \mathrm{g}$ & $480 \mu \mathrm{g}$ & $690 \mu \mathrm{g}$ \\
\hline$N$ & 36 & 34 & 30 \\
Retained volume $(\mu \mathrm{l})$ & $35.1 \pm 8.9^{\mathrm{a}}$ & $34.5 \pm 10.8$ & $39.5 \pm 10.9$ \\
Minimum $(\mu \mathrm{l})$ & 21.4 & 22.1 & 19.7 \\
Maximum $(\mu \mathrm{l})$ & 58.9 & 77.1 & 66.1 \\
\hline
\end{tabular}

Target retained volume $=30 \mu \mathrm{l}$.

a Mean \pm std deviation. 


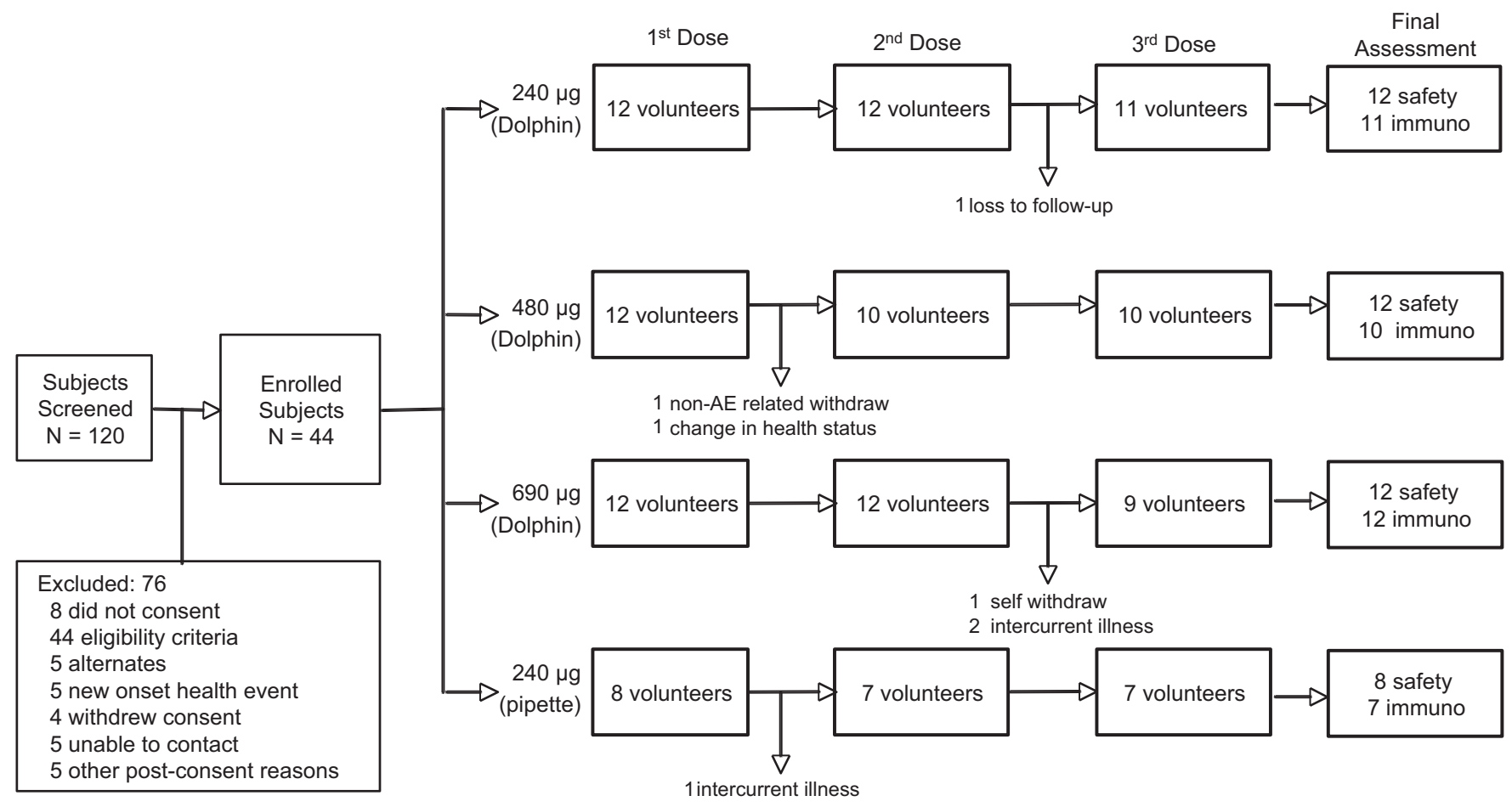

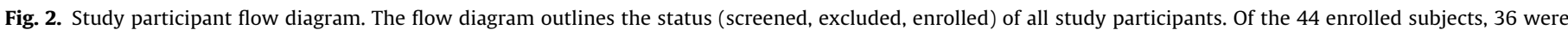

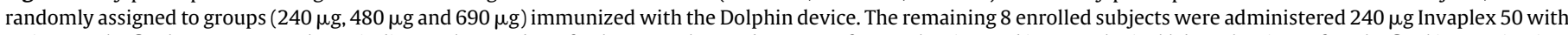

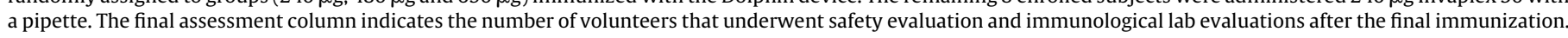

clinical chemistry values. Table 4 details the surveyed signs and symptoms of volunteers receiving at least one vaccine dose. The majority (98\%) of the vaccine-attributable adverse events (AE) were mild, self-limiting, and resolved within $72 \mathrm{~h}$ after immunization. No volunteers reported any vaccine-attributable severe or serious adverse events. The most commonly reported vaccine-associated symptoms were rhinorrhea (31\%) and nasal congestion (33\%) with no significant differences in frequencies between groups. Sneezing and nasal itching were also observed relatively frequently (both in $28 \%$ of the volunteers). The CochranArmitage Chi-square tests for trend of increasing AE frequency with increasing Invaplex 50 dose approached statistical significance for transient nasal itching $(p=0.07)$, coughing $(p=0.08)$ and sneezing $(p=0.07)$. The frequency of headaches, nasal congestion, nasal itching, sore throat and sneezing decreased upon repeated vaccination (Cochran-Armitage Chi-square $p$-value $<0.05$ ) with the most evident decreases occurring between dose 1 and dose 2 .

Adverse events within the first 30 min after vaccine administration were reported and most frequently included sneezing (18.6\%), post-nasal drip (18.6\%), nasal itching (16.3\%) and nasal congestion
(16.3\%). Adverse events were also reported between $24 \mathrm{~h}$ and $72 \mathrm{~h}$ following vaccination and included nasal congestion (32.6\%) and post-nasal drip (16.3\%). Lymphadenopathy (cervical) and pharyngeal erythema were observed on clinical exam and coded as at least possibly related to the investigational product in $14 \%$ and $6 \%$ of volunteers, respectively. The development of mucosal alterations did not occurr during the study. Two cases of subjective fever were reported in the $240 \mu \mathrm{g}$ dose groups delivered with the Dolphin ${ }^{\mathrm{TM}}$ and two cases with the pipette. There were no significant differences in the frequency of any physical exam findings between the vaccine groups. Two possibly related vaccine-attributable moderate adverse events (cough and sore throat) were reported by a single subject in the $690 \mu \mathrm{g}$ dose group just over 1 week after receipt of the initial vaccine dose.

\subsection{Immunological responses}

Of the 33 volunteers that received the minimum number (two) of vaccinations required for immunologic evaluation via the intranasal spray device, 11 were in the $240 \mu \mathrm{g}$ dose group, 10 in the $480 \mu \mathrm{g}$ dose group and 12 in the $690 \mu \mathrm{g}$ dose group. A total

Table 3

Baseline characteristics of study participants.

\begin{tabular}{|c|c|c|c|c|}
\hline Invaplex 50 dose $^{\mathrm{a}}$ & $240 \mu \mathrm{g}$ (Pipette) & $240 \mu \mathrm{g}\left(\right.$ Dolphin $\left.^{\mathrm{TM}}\right)$ & $480 \mu \mathrm{g}\left(\right.$ Dolphin $\left.^{\mathrm{TM}}\right)$ & $690 \mu \mathrm{g}\left(\right.$ Dolphin $\left.{ }^{\mathrm{TM}}\right)$ \\
\hline$N$ & 8 & 12 & 12 & 12 \\
\hline Median (IQR) age & $28.0(24.0,32.0)$ & $41.0(32.5,43.5)$ & $41.5(36.0,42.5)$ & $30.5(24.0,38.0)$ \\
\hline \multicolumn{5}{|l|}{ Gender } \\
\hline$n(\%)$ male & $5(62.5)$ & $7(58.3)$ & $7(58.3)$ & $5(41.7)$ \\
\hline$n(\%)$ female & $3(37.5)$ & $5(41.7)$ & $5(41.7)$ & $7(58.3)$ \\
\hline \multicolumn{5}{|l|}{ Race/ethnicity } \\
\hline$n(\%)$ African-American & $2(25.0)$ & $7(58.3)$ & $8(66.7)$ & $9(75.0)$ \\
\hline$n(\%)$ Caucasian & $3(37.5)$ & $5(41.7)$ & $3(25.0)$ & $1(8.3)$ \\
\hline$n(\%)$ other & $3(37.5)$ & $0(0.0)$ & $1(8.3)$ & $2(16.7)$ \\
\hline
\end{tabular}

$N=$ number receiving at least one immunization; $\mathrm{IQR}=$ interquartile range.

a Dose amount represents total protein. 
Table 4

Number (percent) of volunteers experiencing surveyed signs and symptoms post-vaccination with S. flexneri 2a Invaplex 50.

\begin{tabular}{|c|c|c|c|c|c|c|c|c|}
\hline \multirow[t]{2}{*}{ Adverse event } & \multicolumn{2}{|c|}{$240 \mu \mathrm{g}$ (Pipette) } & \multicolumn{2}{|c|}{$240 \mu g\left(\right.$ Dolphin $\left.^{\mathrm{TM}}\right)$} & \multicolumn{2}{|c|}{$480 \mu \mathrm{g}\left(\right.$ Dolphin $\left.^{\mathrm{TM}}\right)$} & \multicolumn{2}{|c|}{$690 \mu \mathrm{g}\left(\right.$ Dolphin $\left.^{\mathrm{TM}}\right)$} \\
\hline & Related & Unrelated & Related & Unrelated & Related & Unrelated & Related & Unrelated \\
\hline \multicolumn{9}{|l|}{ Solicited symptoms } \\
\hline Malaise & $1(12.5)$ & $3(37.5)$ & $1(8.3)$ & $3(25.0)$ & $0(0.0)$ & $2(16.7)$ & $1(8.3)$ & $2(16.7)$ \\
\hline Headache & $1(12.5)$ & $1(12.5)$ & $1(8.3)$ & $4(33.3)$ & $1(8.3)$ & $1(8.3)$ & $2(16.7)$ & $6(50.0)$ \\
\hline Rhinorrhea & $1(12.5)$ & $2(25.0)$ & $4(33.3)$ & $4(33.3)$ & $3(25.0)$ & $1(8.3)$ & $4(33.3)$ & $4(33.3)$ \\
\hline Nasal congestion & $2(25.0)$ & $4(50.0)$ & $7(58.3)$ & $5(41.7)$ & $2(16.7)$ & $4(33.3)$ & $3(25.0)$ & $7(58.3)$ \\
\hline Nasal burning & $1(12.5)$ & $0(0.0)$ & $1(8.3)$ & $0(0.0)$ & $0(0.0)$ & $0(0.0)$ & $0(0.0)$ & $1(8.3)$ \\
\hline Nasal itching & $1(12.5)$ & $0(0.0)$ & $1(8.3)$ & $1(8.3)$ & $4(33.3)$ & $1(8.3)$ & $5(41.7)$ & $3(25.0)$ \\
\hline Sore throat & $1(12.5)$ & $1(12.5)$ & $2(16.7)$ & $2(16.7)$ & $0(0.0)$ & $1(8.3)$ & $4(33.3)$ & $3(25.0)$ \\
\hline Postnasal drip & $2(25.0)$ & $1(12.5)$ & $2(16.7)$ & $2(16.7)$ & $2(16.7)$ & $1(8.3)$ & $5(41.7)$ & $2(16.7)$ \\
\hline Cough & $0(0.0)$ & $3(37.5)$ & $1(8.3)$ & $3(25.0)$ & $0(0.0)$ & $2(16.7)$ & $4(33.3)$ & $5(41.7)$ \\
\hline Sinus pain & $0(0.0)$ & $1(12.5)$ & $0(0.0)$ & $0(0.0)$ & $0(0.0)$ & $1(8.3)$ & $1(8.3)$ & $2(16.7)$ \\
\hline Sneezing & $0(0.0)$ & $3(37.5)$ & $1(8.3)$ & $2(16.7)$ & $4(33.3)$ & $1(8.3)$ & $5(41.7)$ & $6(50.0)$ \\
\hline Itching eyes & $0(0.0)$ & $0(0.0)$ & $1(8.3)$ & $0(0.0)$ & $1(8.3)$ & $0(0.0)$ & $2(16.7)$ & $1(8.3)$ \\
\hline Nose bleed & $0(0.0)$ & $0(0.0)$ & $0(0.0)$ & $2(16.7)$ & $0(0.0)$ & $0(0.0)$ & $0(0.0)$ & $0(0.0)$ \\
\hline Fever & $0(0.0)$ & $2(25.0)$ & $1(8.3)$ & $2(16.7)$ & $0(0.0)$ & $1(8.3)$ & $1(8.3)$ & $2(16.7)$ \\
\hline \multicolumn{9}{|l|}{ Physical exam findings } \\
\hline Nasal mucosa hyperemia & $1(12.5)$ & $4(50.0)$ & $1(8.3)$ & $8(66.7)$ & $0(0.0)$ & $7(58.3)$ & $0(0.0)$ & $8(66.7)$ \\
\hline Nasal discharge & $1(12.5)$ & $5(62.5)$ & $1(8.3)$ & $9(75.0)$ & $0(0.0)$ & $10(83.3)$ & $0(0.0)$ & $9(75.0)$ \\
\hline Nasal edema & $0(0.0)$ & $4(50.0)$ & $1(8.3)$ & $8(66.7)$ & $0(0.0)$ & $11(91.7)$ & $0(0.0)$ & $12(100)$ \\
\hline Pharyngeal erythema & $0(0.0)$ & $1(12.5)$ & $0(0.0)$ & $2(16.7)$ & $0(0.0)$ & $3(25.0)$ & $2(16.7)$ & $3(25.0)$ \\
\hline Sinus tenderness & $0(0.0)$ & $0(0.0)$ & $0(0.0)$ & $0(0.0)$ & $0(0.0)$ & $0(0.0)$ & $0(0.0)$ & $1(8.3)$ \\
\hline Lymphadenopathy & $0(0.0)$ & $2(25.0)$ & $2(16.7)$ & $0(0.0)$ & $2(16.7)$ & $2(16.7)$ & $1(8.3)$ & $0(0.0)$ \\
\hline Conjunctival injection & $0(0.0)$ & $5(62.5)$ & $0(0.0)$ & $2(16.7)$ & $0(0.0)$ & $1(8.3)$ & $0(0.0)$ & $2(16.7)$ \\
\hline Tearing & $0(0.0)$ & $0(0.0)$ & $0(0.0)$ & $0(0.0)$ & $0(0.0)$ & $0(0.0)$ & $0(0.0)$ & $0(0.0)$ \\
\hline Epistaxis & $0(0.0)$ & $0(0.0)$ & $0(0.0)$ & $1(8.3)$ & $0(0.0)$ & $0(0.0)$ & $0(0.0)$ & $0(0.0)$ \\
\hline Abnormal lung exam finding & $0(0.0)$ & $0(0.0)$ & $0(0.0)$ & $0(0.0)$ & $0(0.0)$ & $0(0.0)$ & $0(0.0)$ & $1(8.3)$ \\
\hline Abnormal cranial nerve finding & $0(0.0)$ & $0(0.0)$ & $0(0.0)$ & $0(0.0)$ & $0(0.0)$ & $0(0.0)$ & $0(0.0)$ & $0(0.0)$ \\
\hline
\end{tabular}

of 7 volunteers received at least two doses of vaccine via pipette (Table 5).

\subsubsection{Antigen-specific ASCs}

LPS and Invaplex 50-specific IgG and IgA ASCs were determined for individual volunteers from PBMCs collected before vaccination and 7 days following each dose. Baseline (day 0) antigen-specific ASCs were at undetectable levels in all but two volunteers with $\leq 3$ per $10^{6}$ PBMCs. Positive antigen-specific ASC responses were detected in volunteers from all study groups. In general, antigenspecific IgA and IgG ASCs peaked following the second vaccination in the majority (75-77\%) of volunteers regardless of vaccine dose used for immunization or the antigen specificity of the assay (data not shown).

The $690 \mu \mathrm{g}$ dose elicited the highest frequency of responders with $75 \%$ and $58 \%$ of volunteers having Shigella-specific IgA and IgG ASC responses to $S$. flexneri 2a Invaplex 50, respectively (Table 5;
Fig. 3A). The majority (58\%) of volunteers immunized with the $690 \mu \mathrm{g}$ dose also had positive IgG and IgA ASC responses to LPS and Invaplex 50. Among volunteers receiving the highest dose $(690 \mu \mathrm{g})$, $75 \%$ had either IgA ASCs or IgG ASCs directed to one of the antigens, although the magnitude of the responses varied (IgA range 10-227; IgG range 11-373). Antigen-specific immune responses elicited after immunization with $690 \mu \mathrm{g}$ dose of Invaplex 50 in each volunteer is summarized in Table 6. In terms of the magnitude of the antigen-specific ASC responses, peak Invaplex 50-specific IgA and IgG ASCs were significantly higher in volunteers immunized with $690 \mu \mathrm{g}$ as compared to 240 or $480 \mu \mathrm{g}$ of the vaccine. Furthermore, several volunteers in the 480 and $690 \mu \mathrm{g}$ dose groups had IgG and IgA-secreting ASCs that exceeded 100 (Fig. 3A). In general, the lower quantity of Invaplex 50 used for immunization resulted in a lower frequency of ASC responders (Table 5) and generally lower magnitude of ASCs in those volunteers that did respond (Fig. 3A) in a dose dependent manner. The responder rate in the $240 \mu \mathrm{g}$ dose

Table 5

Frequency of volunteers ${ }^{\mathrm{a}}$ exhibiting an immune response to each of evaluated parameters.

\begin{tabular}{|c|c|c|c|c|c|}
\hline \multirow[t]{2}{*}{ Antigen } & \multirow[t]{2}{*}{ Assay $^{\mathrm{b}}$} & \multicolumn{4}{|l|}{ Study groups } \\
\hline & & $240 \mu \mathrm{g}$ (Pipette) $(n=7)$ & $240 \mu \mathrm{g}\left(\right.$ Dolphin $\left.^{\mathrm{TM}}\right)(n=11)$ & $480 \mu \mathrm{g}\left(\right.$ Dolphin $\left.^{\mathrm{TM}}\right)(n=10)$ & $690 \mu \mathrm{g}\left(\right.$ Dolphin $\left.^{\mathrm{TM}}\right)(n=12)$ \\
\hline \multirow[t]{6}{*}{ Invaplex 50} & $\operatorname{IgA}(\mathrm{ASCs})$ & $1(14)$ & $2(18)^{c}$ & $2(20)$ & $9(75)$ \\
\hline & $\operatorname{IgG}(\mathrm{ASCs})$ & $1(14)$ & $3(27)$ & $2(20)$ & $7(58)$ \\
\hline & IgA (fecal) & $2(29)$ & $7(64)$ & $6(60)$ & $7(58)$ \\
\hline & IgA (nasal) & 0 & $1(9)$ & $1(10)$ & $4(33)$ \\
\hline & IgA (plasma) & 0 & 0 & $1(10)$ & $6(50)$ \\
\hline & IgG (plasma) & 0 & $3(27)$ & $3(30)$ & $2(17)$ \\
\hline \multirow[t]{6}{*}{ LPS } & $\operatorname{IgA}(\mathrm{ASCs})$ & $1(14)$ & $3(27)$ & $2(20)$ & $7(58)$ \\
\hline & $\operatorname{IgG}(\mathrm{ASCs})$ & $1(14)$ & $4(36)$ & $2(20)$ & $7(58)$ \\
\hline & IgA (fecal) & $1(14)$ & $7(64)$ & $7(70)$ & $6(50)$ \\
\hline & IgA (nasal) & $1(14)$ & $1(9)$ & $2(20)$ & $6(50)$ \\
\hline & IgA (plasma) & 0 & $1(9)$ & $1(10)$ & $3(25)$ \\
\hline & IgG (plasma) & 0 & $5(46)$ & $4(40)$ & $5(42)$ \\
\hline
\end{tabular}

\footnotetext{
a Limited to volunteers providing specimens after receipt of at least 2 vaccine doses.

b Immune response definitions: serologic/fecal/nasal ( $\geq 4$-fold rise from baseline) and ASC ( $\geq 10$ antigen-specific cells per $10^{6} \mathrm{PBMC}$ ).

c Data expressed as the number of volunteers with an immune response (\%).
} 


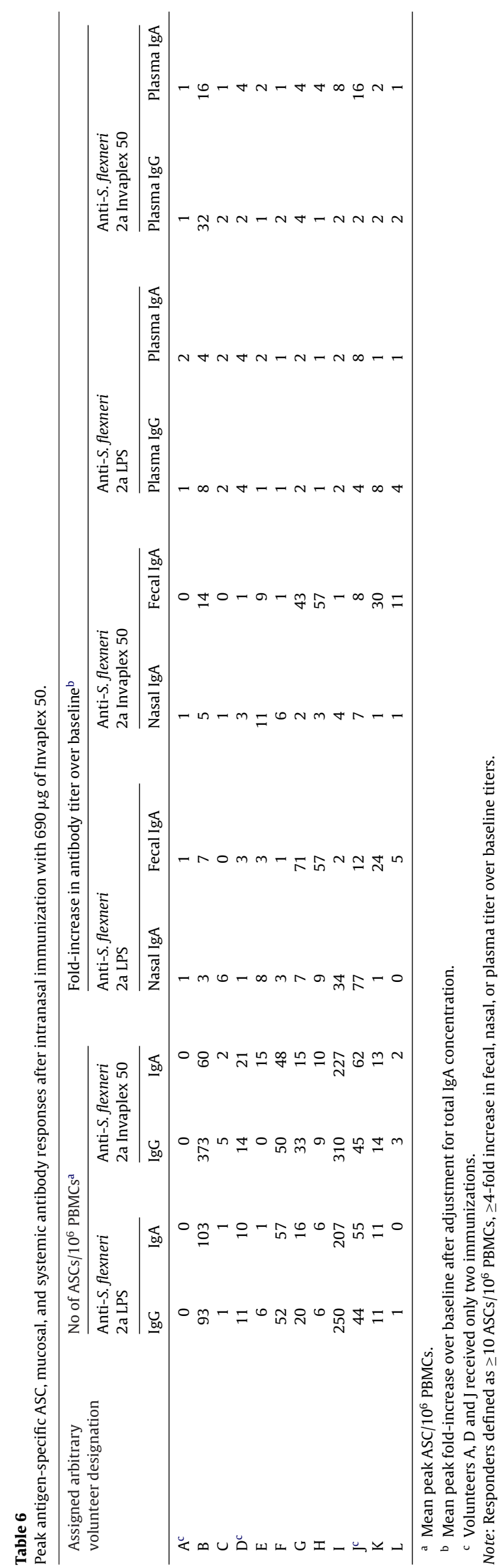

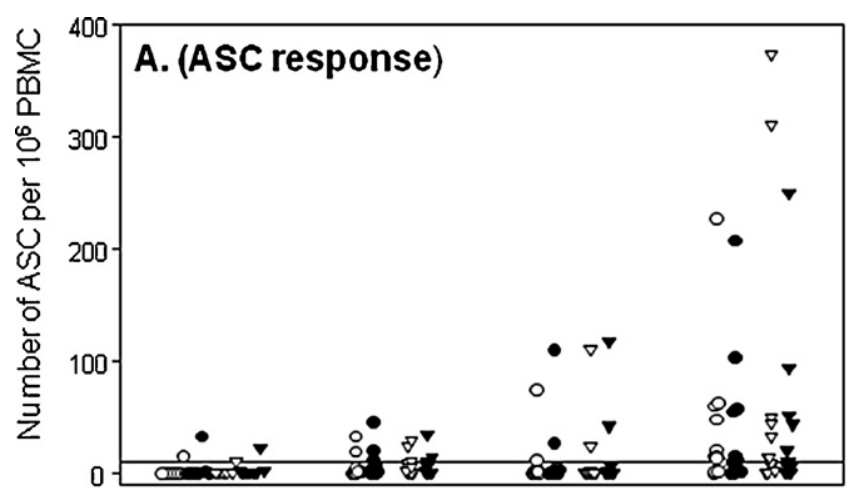
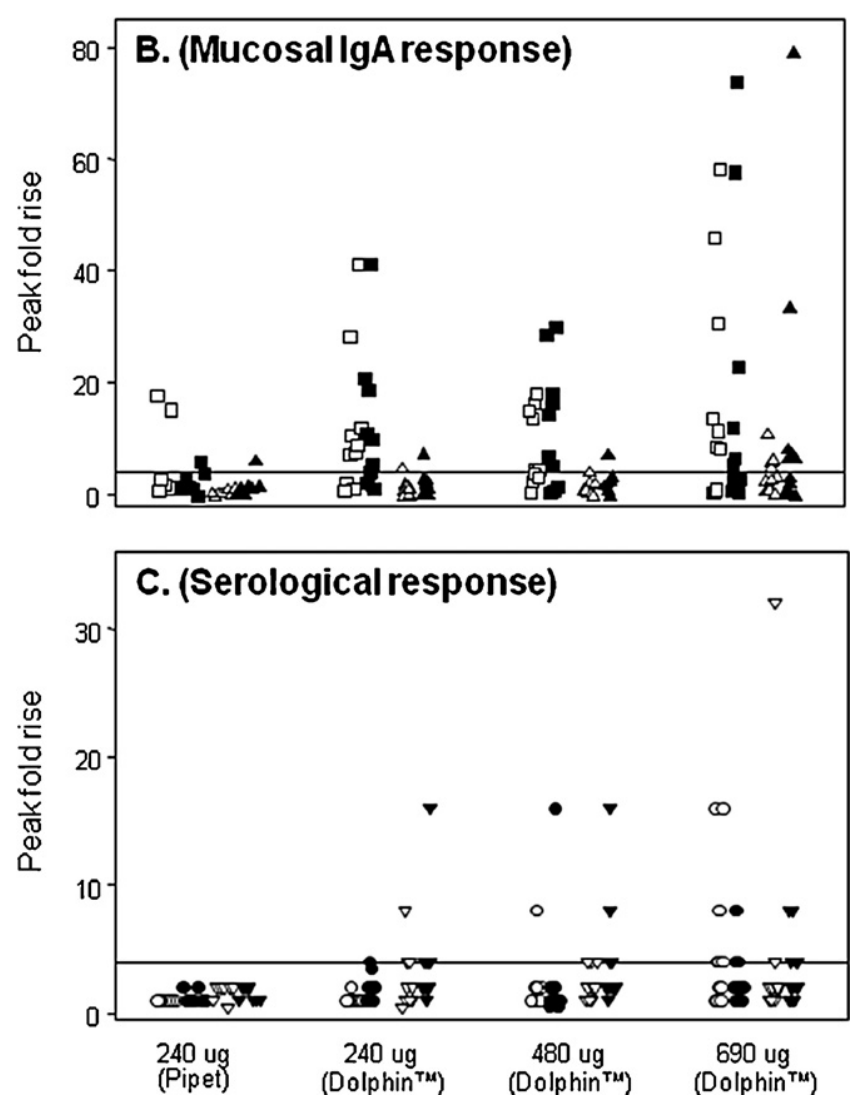

Fig. 3. Peak immune responses after intranasal immunization with $S$. flexneri 2 a Invaplex 50. Panel A: antibody secreting cell responses to Invaplex $50(\operatorname{Ig} A(\bigcirc)$; $\operatorname{IgC}$ $(\nabla))$ and LPS $(\operatorname{IgA}(\bullet)$; $\operatorname{IgG}(\boldsymbol{\nabla}))$. Panel B: mucosal IgA responses to Invaplex 50 (fecal $(\square)$; nasal $(\triangle))$ and LPS (fecal $(\mathbf{\square})$ nasal $(\boldsymbol{\Delta}))$. Panel C: serologic responses to Invaplex $50(\operatorname{IgA}(\bigcirc) ; \operatorname{IgG}(\nabla))$ and LPS $(\operatorname{IgA}(\bullet) ; \operatorname{IgG}(\mathbf{v}))$. Note: The horizontal lines denote the minimum responder level. ASC = antibody secreting cell, $\mathrm{PBMC}=$ peripheral blood mononuclear cell, and LPS = lipopolysaccharide.

group (pipette) was low (14\%) as was the magnitude of the ASC response in the volunteer that responded to the vaccine.

\subsubsection{Fecal and nasal IgA response}

Fecal and nasal samples were collected pre-immunization and on study days 21,35 , and 56 (Fig. 3B). Fecal IgA responder rates were similar across all dose levels ( $\sim 60 \%$ responder rate, Table 5$)$. Among responders, there was a trend towards higher peak foldrises in antigen-specific fecal IgA in the $690 \mu \mathrm{g}$ dose group with mean peak fold increases of 29 (range 5-71) and 25 (range 8-57) for LPS and Invaplex 50-specific IgA, respectively, as compared to 12-17-fold increases over baseline in the 240 and $480 \mu \mathrm{g}$ dose groups for each antigen. Nasal IgA responder rate had an apparent dose-response for LPS $(p=0.03)$ and a similar trend for Invaplex 
$50(p=0.13)$ (Table 5). LPS and Invaplex 50-specific mean peak fold rises among responders in the $690 \mu \mathrm{g}$ dose group were 24 (range 7-77) and 7 (range 6-11), respectively (Table 6). Responder rates in the $240 \mu \mathrm{g}$ (pipette) dose group were low for both Invaplex 50specific fecal (14-29\%) and LPS-specific nasal IgA (14\%).

\subsubsection{Serological responses}

LPS-specific plasma IgG response rates ranged from $40-46 \%$ and Invaplex 50-specific response rates were $17-30 \%$ across all three dose groups receiving the nasal spray (Table 5; Fig. 3C). Only a small percentage $(\leq 25 \%)$ of volunteers had LPS-specific plasma IgA response regardless of vaccine dose. The Invaplex 50-specific plasma IgA response rate ranged from 0 to $50 \%$ with the $690 \mu \mathrm{g}$ dose group having the highest response rate (50\%). No seroconversion was detected after immunization with $240 \mu \mathrm{g}$ delivered intranasally with a pipette.

\subsubsection{Comparison between vaccine delivery methods}

In general, higher ASC, plasma and mucosal immune responses were noted in the group that received the $240 \mu \mathrm{g}$ via intranasal spray compared to those volunteers receiving the same dose by pipette (Table 5; Fig. 3A and B). Specifically, fecal IgA response rates were two-fold higher against Invaplex 50 antigen (64\% versus $29 \%$; $p=0.33$ ) and four-fold higher against LPS (64\% versus $14 \% ; p=0.07$ ). Plasma IgG responses to Invaplex 50 and LPS were not detected in the pipette group, whereas the intranasal spray device group had serological IgG responder rates of $27 \%$ and $46 \%$ to Invaplex 50 and LPS antigens, respectively.

\section{Discussion}

The S. flexneri 2a Invaplex 50 intranasal vaccine was safe and immunogenic in a double-blind, expanded safety and immunogenicity study. The results from the current trial are similar to the results from the initial evaluation of the Invaplex 50 vaccine [16] with respect to the absence of serious adverse events, low frequency of adverse events and the vaccine being well tolerated. In addition, there were several new aspects to the current trial as compared to the previous clinical evaluation. In the current trial, a new cGMP lot of Invaplex 50 manufactured using animal product-free culture media was utilized. The differences in the culture medium components did not impact the cGMP manufacture of the vaccine as similar concentrations of the major vaccine constituents (LPS, IpaB and IpaC) were achieved with Invaplex 50 lot 1307 as compared with Invaplex 50 lot 0994, which was used in the previous trial. Furthermore, both cGMP Invaplex 50 vaccine lots had comparable levels of immunogenicity and protective efficacy in the mouse and guinea pig models.

Another difference in the current trial is the use of a nasal spray device rather than a pipette for delivery of the Invaplex 50 vaccine. To our knowledge, this is the first reported study in which the Dolphin ${ }^{\mathrm{TM}}$ intranasal spray device has been used in a human clinical trial. Our evaluation of this device demonstrated favorable performance characteristics in terms of device mechanical function, product stability within the device, and reproducibility of spray volumes in a clinical research setting. The observed higher immune responses using the spray device compared to nasal pipette delivery suggests that the spray characteristics may have facilitated antigen presentation by enhancing uptake by $\mathrm{M}$ cells and delivery to antigen presenting cells (APCs) of the mucosal immune system [20]. Currently there is a licensed trivalent live-attenuated influenza vaccine delivered by the intranasal route. The product is safe and effective delivered as a large droplet aerosol $(0.2 \mathrm{ml})$ that deposits in the nasopharynx. Compared to parenteral administration of influenza split product vaccines, intranasal delivery of live-attenuated virus has the potential advantage to induce both systemic and broad mucosal immune responses [25]. Despite lower hemagglutination inhibition titers in the intranasally delivered vaccine compared to the intramuscular delivered vaccine [26], they are equally effective in preventing homologous influenza illness associated infections $[27,28]$. Mucosally delivered influenza vaccine also appears to provide evidence of immunity against heterologous virus strains $[26,29]$, and is effective in priming immune responses in young children $[26,30]$.

Compared to the previous study in which the Invaplex 50 vaccine was administered intranasally by pipette, the vaccine performed similarly and was well tolerated in humans when given as an intranasal spray. In contrast to the previous study [16], we noticed a trend towards an increase in local nasal signs and symptoms (sneezing, nasal itching, coughing) with increasing vaccine dose among volunteers, a finding that was reported in a prior dose-escalation study of a proteosome-S. flexneri 2a LPS intranasal vaccine [31]. However, similar to the prior Invaplex study, the symptoms were mild, self-limited and well tolerated. Future studies utilizing a placebo group would be helpful in further evaluating these findings.

There was no significant difference in adverse events between groups immunized with $240 \mu \mathrm{g}$ delivered via pipette or spray device in the current study. However, there was a trend towards higher responder rates when the vaccine was delivered via the Dolphin ${ }^{\mathrm{TM}}$ spray device, mostly notably in the fecal IgA responder rates. Modest increases in the responder rates for antigen-specific plasma and ASC IgG were also achieved when the vaccine was administered as a spray versus droplets with a pipette. Furthermore, increases in the immune responses were evident after immunization with $690 \mu \mathrm{g}$ as compared to 480 or $240 \mu \mathrm{g}$ delivered with the spray device suggesting a dose-response.

In addition to an increase in the responder rate post vaccination, the intranasal Invaplex 50 vaccine induced Shigella-specific immune responses of comparable magnitude and frequency as those immunized with live-attenuated Shigella vaccines [32]. Oral immunization with $1 \times 10^{4}$ of the SC602 vaccine induced LPSspecific IgA and IgG ASCs in $58 \%$ and $42 \%$ of volunteers, respectively, and demonstrated protection against experimental challenge with S. flexneri 2a $2457 \mathrm{~T}\left(2 \times 10^{3} \mathrm{cfu}\right)$ among individuals with peak ASC values greater than 75 per $10^{6}$ PBMC. In the current study, intranasal immunization with Invaplex $50(690 \mu \mathrm{g})$ induced LPSspecific ASCs in $58 \%$ of the volunteers. The magnitude of the ASC responses induced after immunization with the oral SC602 vaccine and Invaplex 50 vaccine were also similar with $33 \%$ of the volunteers having $a \geq 50 \operatorname{IgA} A S C / 10^{6}$ PBMCs in each study. Although the numbers of volunteers in each study were low, the data are encouraging especially in light of the efficacy results in the SC602 study.

An equally important finding in the current study is the induction of strong mucosal IgA responses in the gastrointestinal tract evidenced by antigen-specific fecal IgA and ASCs detected after intranasal immunization with Invaplex 50. Presumably, when PBMCs are collected from systemic circulation, ASCs are captured as they were transiting from inductive sites to distal mucosal effector sites. The homing potential of lymphocyte subsets is largely dependent on the expression of key cell adhesion molecules. For mucosal homing, it is widely accepted that $\alpha 4 \beta 7$ on lymphocytes is critical for entry into the gut mucosa [33]. Although the present study has not specifically addressed the expression of homing receptors on lymphocytes collected after intranasal immunization, the antigenspecific IgA ASCs and fecal IgA responses detected after intranasal immunization with Invaplex 50 suggest that lymphocytes are homing to the gut, perhaps similar to what has recently been described for a Norwalk virus-like particle (VLP) vaccine [34]. In that study, intranasally administered Norwalk VLPs induced high levels of IgA ASCs. The homing receptors on the IgA ASCs supported homing 
to the gut mucosa $(\alpha 4 \beta 7+/ C D 62 \mathrm{~L}-)$ whereas VLP-specific IgG ASCs expressed receptors indicative of homing to mesenteric and peripheral lymphoid tissues $(\alpha 4 \beta 7+/ C D 62 \mathrm{~L}+)$. Furthermore, intranasal immunization with Norwalk VLPs was unable to induce an immune response in which the lymphocytes would home exclusively to peripheral lymphoid tissues ( $\alpha 4 \beta 7-/ C D 62 \mathrm{~L}+$ ). These findings may help explain the low level of LPS and Invaplex 50-specific IgA and IgG in the plasma after intranasal immunization with Invaplex 50. Additional studies are required to determine if IgA ASCs induced after Invaplex 50 immunization possess similar mucosal homing adhesion molecules.

With respect to development of a Shigella vaccine, the ability of a mucosally delivered subunit protein LPS complex vaccine to induce both systemic and mucosal immune response would have certain advantages in targeting colonization and invasion related to Shigella pathogenesis. Nasal immunization with the Invaplex 50 product in clinical setting indicates that the Invaplex protein subunit/LPS complex vaccine is well tolerated. In the current study, the upper dose $(690 \mu \mathrm{g})$ contained $113 \mu \mathrm{g}$ of LPS in complex with IpaB and IpaC proteins. It would appear that the dose of Invaplex 50 could be increased to attain a more potent immune response given that $S$. flexneri 2a LPS has been given intranasally in proteosomes at dose amounts of $1.34 \mathrm{mg}$ in a previous clinical trial without serious adverse events [31]. However, a more concentrated Invaplex 50 vaccine would have to be manufactured as the maximum dose $(690 \mu \mathrm{g})$ was given in the current trial due to restrictions on the maximum volume $(100 \mu \mathrm{l})$ delivered by the Dolphin $^{\mathrm{TM}}$ device. Finally, there are practical advantages to nonneedle delivery of vaccines (including intranasal delivery) in terms of reducing the risks of needle stick injuries, as well as perhaps being more acceptable to patients with aichmophobia. These perceived benefits would need to be weighed with the potential extra cost associated with device manufacture and packaging.

The results from this trial are encouraging and provide the necessary data to advance the development of the Invaplex 50 vaccine. The acceptable safety profile combined with the robust immune response demonstrated at the $690 \mu \mathrm{g}$ dose warrants moving forward into a phase $2 \mathrm{~b}$ challenge study (proof of concept) to evaluate vaccine efficacy, as well as expanded information on immunogenicity and safety in a placebo-controlled study. If efficacious, development of a multivalent Shigella vaccine capable of providing immunological protection against S. flexneri 2a, S. sonnei, and $S$. dysenteriae 1 will be developed utilizing current manufacturing processes would proceed towards field testing. Concurrently, enhanced immunogenicity may be achieved in the clinic with second generation Invaplex 50 vaccines assembled from purified, recombinant IpaB and IpaC and phenol-extracted LPS as has been the case in pre-clinical evaluations in the mouse and guinea pig models (Oaks and Kaminski, unpublished results). Further studies evaluating vaccine dosing and regimens will also be required as these products move through the vaccine development pipeline.

\section{Acknowledgements}

Human Volunteers Protection: This study was approved by the ethical review committees of the Naval Medical Research Center, Silver Spring, MD (DoD \#11615, WRAIR 1328, HSRRB Log No. A14057) in compliance with all Federal regulations governing the protection of human volunteers and registered on ClinicalTrials.gov (NCT00485134).

Copyright statement: Authors are military service members or employees of the U.S. Government. This work was prepared as part of official duties. Title 17 U.S.C. $§ 105$ provides that 'Copyright protection under this title is not available for any work of the United States Government.' Title 17 U.S.C. §101 defines a U.S. Government work as a work prepared by a military service member or employee of the U.S. Government as part of that person's official duties

Funding: This study was conducted under support of Military Infectious Disease Research Program.

\section{References}

[1] Kotloff KL, Winickoff JP, Ivanoff B, Clemens JD, Swerdlow DL, Sansonetti PJ, et al. Global burden of Shigella infections: implications for vaccine development and implementation of control strategies. Bull WHO 1999;77:651-66.

[2] Black RE. Epidemiology of travelers' diarrhea and relative importance of various pathogens. Rev Infect Dis 1990;12(January-February (Suppl. 1)):S73-9.

[3] Riddle MS, Sanders JW, Putnam SD, Tribble DR. Incidence, etiology, and impact of diarrhea among long-term travelers (US military and similar populations): a systematic review. Am J Trop Med Hyg 2006;74(May (5)):891-900.

[4] Sharp TW, Thornton SA, Wallace MR, Defraites RF, Sanchez JL, Batchelor RA, et al. Diarrheal disease among military personnel during Operation Restore Hope, Somalia, 1992-1993. Am J Trop Med Hyg 1995;52(February (2)):188-93.

[5] Thornton SA, Sherman SS, Farkas T, Zhong W, Torres P, Jiang X. Gastroenteritis in US marines during Operation Iraqi Freedom. Clin Infect Dis 2005;40(February (4)):519-25.

[6] Wang LH, Fang XC, Pan GZ. Bacillary dysentery as a causative factor of irritable bowel syndrome and its pathogenesis. Gut 2004;53(August (8)):1096-101.

[7] Hannu T, Mattila L, Siitonen A, Leirisalo-Repo M. Reactive arthritis attributable to Shigella infection: a clinical and epidemiological nationwide study. Ann Rheum Dis 2005;64(April (4)):594-8.

[8] Kaminski RW, Oaks EV. Inactivated and subunit vaccines to prevent shigellosis. Expert Rev Vaccines 2009;8(December (12)):1693-704.

[9] Kweon MN. Shigellosis: the current status of vaccine development. Curr Opin Infect Dis 2008;21(June (3)):313-8.

[10] Levine MM, Kotloff KL, Barry EM, Pasetti MF, Sztein MB. Clinical trials of Shigella vaccines: two steps forward and one step back on a long, hard road. Nat Rev Microbiol 2007;5(July (7)):540-53.

[11] Phalipon A, Mulard LA, Sansonetti PJ. Vaccination against shigellosis: is it the path that is difficult or is it the difficult that is the path? Microbes Infect 2008;10(July (9)):1057-62.

[12] Menard R, Sansonetti PJ, Parsot C. Nonpolar mutagenesis of the ipa genes defines IpaB, IpaC, and IpaD as effectors of Shigella flexneri entry into epithelial cells. J Bacteriol 1993:175:5899-906.

[13] Blocker A, Gounon P, Larquet E, Niebuhr K, Cabiaux V, Parsot C, et al. The tripartite type III secreton of Shigella flexneri inserts IpaB and IpaC into host membranes. J Cell Biol 1999;147:683-93.

[14] Turbyfill KR, Hartman AB, Oaks EV. Isolation and characterization of a Shigella flexneri invasin complex subunit vaccine. Infect Immun 2000;68:6624-32.

[15] Oaks EV, Turbyfill KR. Development and evaluation of a Shigella flexneri 2a and S. sonnei bivalent invasin complex (Invaplex) vaccine. Vaccine 2006;24(March (13)):2290-301.

[16] Tribble D, Kaminski R, Cantrell J, Nelson M, Porter C, Baqar S, et al. Safety and immunogenicity of a Shigella flexneri 2a Invaplex 50 intranasal vaccine in adult volunteers. Vaccine 2010;28(August (37)):6076-85.

[17] Mills JA, Buysse JM, Oaks EV. Shigella flexneri invasion plasmid antigens B and C: epitope location and characterization with monoclonal antibodies. Infect Immun 1988;56(November (11)):2933-41.

[18] Hartman AB, Verg LLVD, Mainhart CR, Tall BD, Smith-Gill SJ. Specificity of monoclonal antibodies elicited by mucosal infection of BALB/c mice with virulent Shigella flexneri 2a. Clin Diag Lab Immunol 1996;3:584-9.

[19] Turbyfill KR, Kaminski RW, Oaks EV. Immunogenicity and efficacy of highly purified invasin complex vaccine from Shigella flexneri 2a. Vaccine 2008;26(March (10)):1353-64

[20] Davis SS. Nasal vaccines. Adv Drug Deliv Rev 2001;51(1-3):21-42.

[21] Carter JD. Reactive arthritis: defined etiologies, emerging pathophysiology, and unresolved treatment. Infect Dis Clin North Am 2006;20(December (4)):827-47.

[22] Granfors K, Jalkanen S, Toivanen P, Koski J, Lindberg AA. Bacterial lipopolysaccharide in synovial fluid cells in Shigella triggered reactive arthritis. J Rheumatol 1992;19(March (3)):500.

[23] Kaminski RW, Turbyfill KR, Oaks EV. Mucosal adjuvant properties of the Shigella invasin complex. Infect Immun 2006;74(May (5)):2856-66.

[24] Baqar S, Applebee LA, Gilliland Jr TC, Lee LH, Porter CK, Guerry P. Immunogenicity and protective efficacy of recombinant Campylobacter jejuni flagellum-secreted proteins in mice. Infect Immun 2008;76(July (7)):3170-5.

[25] Belshe R, Lee MS, Walker RE, Stoddard J, Mendelman PM. Safety, immunogenicity and efficacy of intranasal, live attenuated influenza vaccine. Expert Rev Vaccines 2004;3(December (6)):643-54.

[26] Cox RJ, Brokstad KA, Ogra P. Influenza virus: immunity and vaccination strategies. Comparison of the immune response to inactivated and live, attenuated influenza vaccines. Scand J Immunol 2004;59(January (1)):1-15.

[27] Clements ML, Betts RF, Tierney EL, Murphy BR. Serum and nasal wash antibodies associated with resistance to experimental challenge with influenza A wildtype virus. J Clin Microbiol 1986;24(July (1)):157-60.

[28] Beyer WE, Palache AM, de Jong JC, Osterhaus AD. Cold-adapted live influenza vaccine versus inactivated vaccine: systemic vaccine reactions, local and systemic antibody response, and vaccine efficacy. A meta-analysis. Vaccine 2002;20(January (9-10)):1340-53. 
[29] Lee LY, Ha do LA, Simmons C, de Jong MD, Chau NV, Schumacher R, et al. Memory $\mathrm{T}$ cells established by seasonal human influenza A infection cross-react with avian influenza A (H5N1) in healthy individuals. J Clin Invest 2008;118(October (10)):3478-90.

[30] Fleming DM, Crovari P, Wahn U, Klemola T, Schlesinger Y, Langussis A, et al. Comparison of the efficacy and safety of live attenuated cold-adapted influenza vaccine, trivalent, with trivalent inactivated influenza virus vaccine in children and adolescents with asthma. Pediatr Infect Dis J 2006;25(October (10)): 860-9.

[31] Fries LF, Montemarano AD, Mallett CP, Taylor DN, Hale TL, Lowell GH. Safety and immunogenicity of a proteosome-Shigella flexneri 2a lipopolysaccharide vaccine administered intranasally to healthy adults. Infect Immun 2001;69(July (7)):4545-53.
[32] Coster TS, Hoge CW, VanDeVerg LL, Hartman AB, Oaks EV, Venkatesan MM, et al. Vaccination against Shigellosis with attenuated Shigella flexneri 2a strain SC602. Infect Immun 1999;67:3437-43.

[33] Brandtzaeg P, Johansen FE. Mucosal B cells: phenotypic characteristics, transcriptional regulation, and homing properties. Immunol Rev 2005;206(August):32-63.

[34] El-Kamary SS, Pasetti MF, Mendelman PM, Frey SE, Bernstein DI, Treanor JJ, et al. Adjuvanted intranasal Norwalk virus-like particle vaccine elicits antibodies and antibody-secreting cells that express homing receptors for mucosal and peripheral lymphoid tissues. J Infect Dis 2010;202(December (11)):1649-58.

[35] Hartman AB, Powell CJ, Schultz CL, Oaks EV, Eckels KH. Small-animal model to measure efficacy and immunogenicity of Shigella vaccine strains. Infect Immun 1991;59(11):4075-83. 